\title{
Preface of the Special Issue New Horizons in Optimal Control- a Special Tribute to Helmut Maurer, Urszula Ledzewicz and Heinz Schättler
}

\author{
Regina Burachik ${ }^{1}$ - Maria do Rosário de Pinho $^{2}$ - Claudia Sagastizábal ${ }^{3}$. \\ Hasnaa Zidani ${ }^{4}$
}

Published online: 23 May 2019

(C) Springer Nature B.V. 2019

This is a special issue honoring three distinguished and highly reputed researchers: Urszula Ledzewicz, Helmut Maurer and Heinz Schättler. Each of them, in their own way, has vastly contributed for the development of the field of Optimal Control theory and its application to real life problems.

Helmut Maurer, Urszula Ledzewicz and Heinz Schättler have different backgrounds. Both Helmut and Heinz were born and educated in Germany. While Helmut remained in Germany throughout his career, Heinz headed to the US early in his life, where he got his $\mathrm{PhD}$ and where he has lived since then. Urszula Ledzewicz was born and educated in Poland. In the late 80 's she moved to the US. Not surprisingly, her mathematical roots are in the Russian school.

Helmut Maurer has always looked at any problem from three different sides: development of theory, applications and numerical methods. So, each piece of theory could not rest in peace before being tested in applications and some specific problems being solved numerically. He has offered us over the years important contributions to optimality conditions for constrained optimal control problems and to numerical methods for optimal control.

Urszula Ledzewicz had first produced some impressive results on problems with mixed constraints in her early years while Heinz Schättler followed a Geometric Control approach to optimal control. They both turned to applications and, together, Urszula and Heinz broke new ground on optimal control of biomathematical problems, specially on the study of cancer treatment. They, many times together with Helmut, have shed a new light upon these problems.

Maria do Rosário de Pinho

mrpinho@fe.up.pt

1 University of South Australia, Mawson Lakes, Australia

2 Faculdade de Engenharia, DEEC, Systec, University of Porto, Rua Dr. Roberto Frias s/n, 4200-465 Porto, Portugal

3 DIM and CMM, University of Chile, Santiago, Region Metropolitana, Chile

4 Ecole Nationale Supérieure de Techniques Avancées, Paris, France 
United by their interest on theory and applications of optimal control, Helmut, Urszula and Heinz have been a beacon and a helping hand to many of us in the optimal control community. They have guided and enthusiastically encouraged many researchers in pursuing their work in such an extraordinary field.

These three outstanding researchers have marked with their knowledge, insight and kindness the lives of many of us. This special issue is a simple tribute to their lives and work. It came to be as a result of the conference New Horizons in Optimal Control 2017, held at the University of Porto, Faculdade de Engenharia, Portugal, in July of 2017. It brings together the following papers, on several topics related to optimal control and close fields.

- Sankalp Bhan and Heinz Schättler study the solutions of optimal control problems in $A$ Variational Approach to Perturbation Feedback Control for Optimal Control Problems with Terminal Constraints and Free Terminal Time.

- Sabine Pickenhain concentrates on Infinite Horizon Problems in the Calculus of Variations, The Role of Transformations with an Application to the Brachistochrone Problem.

- Optimal control problems involving partial differential equations are considered in Analysis of Optimal Control Problems of Semilinear Elliptic Equations by BVFunctions by Eduardo Casas.

- Jakob Preininger studies linear quadratic optimal control problems in Metric Regularity Properties in Bang-Bang Type Linear-Quadratic Optimal Control Problems.

- Numerical methods for differential-algebraic systems are treated in Convergence Analysis of the Implicit Euler-discretization and Sufficient Conditions for Optimal Control Problems Subject to Index-one Differential-algebraic Equations by Björn Martens and Matthias Gerdts.

- Carlos Rautenberg and Michael Hintermüller introduce dedicated numerical methods for Dissipative and Non-Dissipative Evolutionary Quasi-Variational Inequalities with Gradient Constraints.

- The study of stability of solutions for problems containing a non-differentiable Nemytskii operator is the subject of Sensitivity Analysis for a Class of $H_{0}^{1}$-Elliptic Variational Inequalities of the Second Kind by Christof Constanti and Christian Meyer.

- State-constrained problems are the focus of attention in Normality of Necessary Optimality Conditions for Calculus of Variations Problems with State Constraints by Nathalie Khalil and Sofia Lopes

- Necessary optimality conditions for a class of problems involving sweeping processes are derived by M. Margarida A. Ferreira, M. Rosario do Pinho and Georgi Smirnov in Optimal Control Involving Sweeping Processes.

- Samir Adly and Taron Zakaryan consider parametric sweeping processes in Sensitivity properties of parametric nonconvex evolution inclusions with application to optimal control problems.

- Emilio Vilches studies a class of sweeping processes in Hilbert spaces in Existence and Lyapunov Pairs for the Perturbed Sweeping Process Governed by a Fixed Set, characterizatizing Lyapunov nonsmooth pairs under general hypotheses.

- Lyapunov pairs in Banach spaces are the topic of Lyapunov pairs in semilinear differential problems with state-dependent impulses by Irene Benedetti, Tiziana Cardinali, Grzegorz Gabor and Paola Rubbioni. 
This volume is a small token of gratitude to three special researchers Helmut Maurer, Urszula Ledzewicz and Heinz Schättler for their dedication, hard work and enthusiasm. We hope that the papers gathered in this special issue may also be inspirational to many.

Regina Burachik, Maria do Rosário de Pinho (Guest Editor), Claudia Sagastizábal and Hasnaa Zidani

Publisher's Note Springer Nature remains neutral with regard to jurisdictional claims in published maps and institutional affiliations. 